\title{
Pralatrexate as a bridge to allogeneic hematopoietic stem cell transplantation in a patient with advanced-stage extranodal nasal-type natural killer/T cell lymphoma refractory to first-line chemotherapy: a case report
}

Yao-Chung Liu ${ }^{1,2^{*}}$, Ting-An Lin ${ }^{1,2}$, Hao-Yuan Wang ${ }^{1,2}$, Po-Shen Ko ${ }^{1,2}$, Chia-Jen Liu ${ }^{1,2}$, Liang-Tsai Hsiao ${ }^{1,2}$, Sheng-Hsuan Chien ${ }^{2,3}$ and Jyh-Pyng Gau ${ }^{1,2}$

\begin{abstract}
Background: Extranodal natural killer/T cell lymphoma, nasal type, is one of the more common subtypes of mature T cell lymphoma, especially in the Far East Asian population. This aggressive histologic subtype of peripheral $T$ cell lymphomas is frequently susceptible to exposure of Epstein-Barr virus infection. The optimal treatment is not well elucidated. For stage IV disseminated extranodal natural killer/T cell lymphoma, induction chemotherapy with consolidative autologus or allogeneic hematopoietic stem cell transplantation is recommended as the major firstline treatment. However, there is controversy over which type of chemotherapy is most appropriate and effective as a bridge to autologus or allogeneic hematopoietic stem cell transplantation in patients with newly diagnosed disseminated advanced-stage or relapsed extranodal natural killer/T cell lymphoma because of cancer chemoresistance or associated complications. Pralatrexate is the first US Food and Drug Administration-approved novel agent for the treatment of refractory/recurrent peripheral T cell lymphomas. In our case, pralatrexate was used as a successful bridge to allogeneic hematopoietic stem cell transplantation in a patient with advanced-stage disseminated extranodal natural killer/T cell lymphoma refractory to first-line chemotherapy.

(Continued on next page)
\end{abstract}

\footnotetext{
*Correspondence: ycliu17@vghtpe.gov.tw

'Division of Haematology, Department of Medicine, Taipei Veterans General

Hospital, No. 201, Section 2, Shipai Road, Beitou District, Taipei City 112,

Taiwan

${ }^{2}$ Faculty of Medicine, National Yang-Ming University, No. 155, Section 2,

Linong St, Beitou District, Taipei City 112, Taiwan

Full list of author information is available at the end of the article
}

C C The Author(s). 2020 Open Access This article is licensed under a Creative Commons Attribution 4.0 International License, which permits use, sharing, adaptation, distribution and reproduction in any medium or format, as long as you give appropriate credit to the original author(s) and the source, provide a link to the Creative Commons licence, and indicate if changes were made. The images or other third party material in this article are included in the article's Creative Commons licence, unless indicated otherwise in a credit line to the material. If material is not included in the article's Creative Commons licence and your intended use is not permitted by statutory regulation or exceeds the permitted use, you will need to obtain permission directly from the copyright holder. To view a copy of this licence, visit http://creativecommons.org/licenses/by/4.0/. The Creative Commons Public Domain Dedication waiver (http://creativecommons.org/publicdomain/zero/1.0/) applies to the data made available in this article, unless otherwise stated in a credit line to the data. 
(Continued from previous page)

Case presentation: We presented a case report of a 29-year-old Asian man diagnosed as having stage IV disseminated extranodal natural killer/T cell lymphoma, nasal type, with skin and bone marrow involvement, whose disease was primary refractory to first-line dexamethasone, methotrexate, ifosfamide, L-asparaginase, and etoposide chemotherapy, but obviously responded to treatment with two cycles of single-agent pralatrexate treatment. Monitoring Epstein-Barr virus viremia revealed dramatic downregulation. In addition to complete remission of the involvement of bone marrow and nasal cavity, skin involvement also obtained partial remission. The extranodal natural killer/T cell lymphoma successfully achieved complete remission after a bridge to allogeneic hematopoietic stem cell transplantation.

Conclusions: This is the first study to present pralatrexate as a successful bridge to allogeneic hematopoietic stem cell transplantation in a 29-year-old Asian male patient with advanced-stage extranodal natural killer/T cell lymphoma refractory to first-line dexamethasone, methotrexate, ifosfamide, L-asparaginase, and etoposide chemotherapy. This case provides a novel treatment opinion for extranodal natural killer/T cell lymphoma, especially for the Far East Asian population.

Keywords: Natural killer/T cell lymphoma, Peripheral T cell lymphoma, Chemotherapy, Pralatrexate, Allogeneic hematopoietic stem cell transplantation

\section{Background}

Natural killer/T cell lymphoma (NKTCL) is one of the more common subtypes of mature $\mathrm{T}$ cell lymphoma, an aggressive subtype of peripheral T cell lymphoma (PTCL), which occurs predominantly in non-nodal sites $[1,2]$. Most extranodal natural killer (NK)/T cell lymphomas (ENKL), nasal type, frequently involve the skin, soft tissue, aerogastrointestinal tract, and testis [1,2]. ENKL is associated with Epstein-Barr virus (EBV)-positive infiltration in lymphoid tissue or viremia and occurs more commonly in Asian and South American populations than in Western populations [2-4]. The quantification of circulating plasma EBV DNA can be used to monitor the treatment response of ENKL [2-4].

Not only do patients with ENKLs have a poor survival rate at 5 years, ranging from 37.9 to $45.3 \%$, but ENKLs also show easy resistance to chemotherapy $[5,6]$. Because ENKL is often resistant to anthracycline-based chemotherapy, regimens based on asparaginase and PEGasparaginase, such as the dexamethasone, methotrexate, ifosfamide, L-asparaginase, and etoposide (SMILE) regimen, have been effective as first-line therapy for nasal type ENKL $[7,8]$. The outcomes for advanced or relapsed/refractory ENKL remain poor, however, particularly once the disease becomes refractory to first-line SMILE chemotherapy. The survival of patients receiving second-line therapy averages less than 6 months $[9,10]$. Pralatrexate (PDX) is a novel drug approved by the US Food and Drug Administration for the treatment of relapsed or refractory PTCL [11]. PDX was developed as a synthetic antimetabolite folate analog and had a higher potency than methotrexate in competitively inhibiting dihydrofolate reductase. The recommended dose of PDX is $30 \mathrm{mg} / \mathrm{m}^{2}$ once weekly for 6 weeks in a 7 -week cycle until disease progresses or there is unacceptable toxicity for PTCL that may require dose reduction or discontinuation [12].
Allogeneic hematopoietic stem cell transplantation (AHSCT) can be used as a first-line treatment to prevent relapse of advanced ENKL or as salvage treatment after chemotherapy [13, 14]. Guidelines from the American Society for Blood and Marrow Transplantation supported the use of both autologous hematopoietic stem cell transplantation (HSCT) and AHSCT for chemosensitive relapsed disease in localized ENKL or as a front-line consolidation therapy for disseminated ENKL [15]. However, there is controversy over which type of HSCT is most appropriate in patients with advanced-stage or relapsed ENKL. For disseminated ENKL with bone marrow involvement, it is very difficult to collect enough autologous stem cells or avoid cancer stem cell harvesting. Until now, though, there remains a lack of effective first-line treatments for newly diagnosed ENKL or a bridge to AHSCT for relapsed/refractory ENKL. PDX provides a new option for salvage therapy or a bridge to AHSCT in patients with refractory or relapsed ENKL. Here we report what we believe to be the first successful use of PDX as a bridge to AHSCT for the treatment of a patient with advanced-stage disseminated ENKL, nasal type, which was refractory to first-line SMILE chemotherapy.

\section{Case presentation}

A 29-year-old Asian man experienced rhinorrhea, intermittent fever up to $40^{\circ} \mathrm{C}\left(104^{\circ} \mathrm{F}\right)$ two to three times per week, and multiple progressive non-itchy papules on his face and trunk since September 2015. He was treated for chronic atopic dermatitis and chronic sinusitis without obvious improvement after multiple courses of antibiotics since April 2016.

In February 2017, a nasopharyngoscopy revealed necrotic tissue in bilateral nasal cavities and septal perforation. Biopsies were completed for both nasal and skin lesions. The pathology of the skin lesions revealed many 
small lymphocytic infiltrates in the perivascular area with positive EBV-encoded small RNA (EBER) stain. The scattered EBER-positive small lymphocytes were also positive for CD3, CD56, and TIA-1 stains and negative for CD20. Numerous reactive $\mathrm{T}$ cells were also noted to be positive: $\mathrm{CD} 2, \mathrm{CD} 3, \mathrm{CD} 4, \mathrm{CD} 5, \mathrm{CD} 7$, and CD8 (Fig. 1); nasal lesions showed a similar picture. A bone marrow aspirate and biopsy showed 20\% small lymphoid cells with special stains similar to skin lesions. A whole-body positron emission tomography (PET)/ computed tomography (CT) scan revealed increased fluorodeoxyglucose (FDG) uptake in his bilateral nasal cavity, bilateral neck level I and II lymph nodes, and bilateral axillary regions. EBV polymerase chain reaction (PCR) for peripheral blood showed a viral load of 15,174 copies/mL. He was diagnosed as having ENKL, nasal type, with an Ann Arbor clinical stage of IV.

In March 2017, he underwent systemic chemotherapy with the SMILE regimen (etoposide $100 \mathrm{mg} / \mathrm{m}^{2}$ on days 2-4; ifosfamide $1500 \mathrm{mg} / \mathrm{m}^{2}$ on days $1-3$; mesna 900 $\mathrm{mg} / \mathrm{m}^{2}$ on days $2-4$; methotrexate $2000 \mathrm{mg} / \mathrm{m}^{2}$ on day 1 ; dexamethasone $40 \mathrm{mg}$ on days $1-4$; and L-asparaginase $6000 \mathrm{U} / \mathrm{m}^{2}$ on days $8,10,12,14,16,18$, and 20 ). Cycles were repeated every 28 days. He received two cycles of SMILE, each complicated by intermittent fever after neutropenic recovery, progressive skin lesions, and rebounding EBV viremia during the chemotherapy window. At this point, his ENKL was noted to be primary refractory to the SMILE regimen; therefore, he received two cycles of PDX $30 \mathrm{mg} / \mathrm{m}^{2}$ on days $1,8,15,22,29$, and 36 in a PDX clinical trial. After two cycles of PDX, a partial remission of skin lesions and complete remission of nasal lesions, neck lymphadenopathies, and bone marrow were documented.

He then underwent a matched unrelated AHSCT. After AHSCT, his disease achieved complete remission. Follow-up of EBV viremia showed significant decrease (Fig. 2). The skin lesions on our patient's face and trunk also revealed complete remission (Fig. 3). A follow-up PET-CT scan of his nasal lesions and lymphadenopathies showed complete metabolic response after one cycle of PDX and maintained complete response (CR) after AHSCT (Fig. 4). He suffered from progressive acute graft-versus-host disease (aGVHD) with overall grade III and refractory to steroid ( $2 \mathrm{mg} / \mathrm{kg}$ per day) since January 2018. After we administered tocilizumab and sirolimus to our patient, aGVHD was gradually controlled. However, local recurrence of left nasal cavity without new lymphadenopathy, skin involvement, and bone marrow invasion was noticed in March 2018. Local radiotherapy with $5000 \mathrm{cGy} / 25$ fractions to the relapse lesion and $4500 \mathrm{cGy} / 25$ fractions to bilateral nasal cavities were administered. PET-CT revealed complete remission of nasal cavity in September 2018. However, one ulcerative skin lesion on his right forearm was noted. A biopsy showed ENKL, nasal type. Therefore, concurrent chemoradiotherapy (CCRT) with gemcitabine, Leunase (Lasparaginase), and oxaliplatin (GeLOX) regimen for four cycles and local radiotherapy with 5000 cGy/20 fractions to his right forearm lesion were administered until
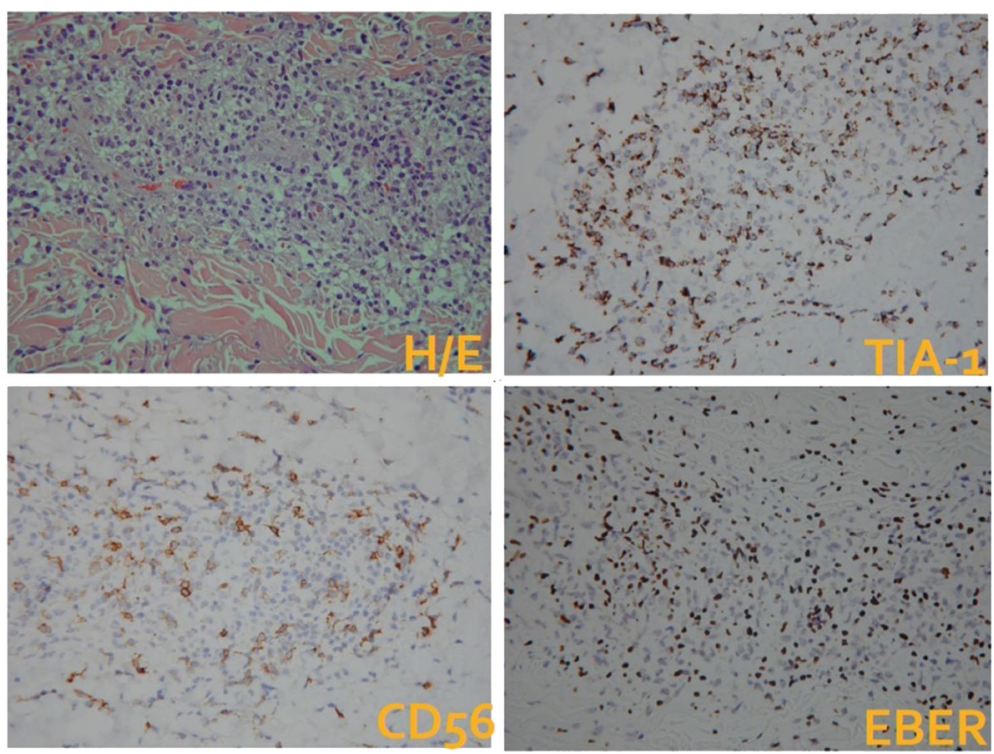

Fig. 1 The scattered Epstein-Barr virus-encoded small RNA-positive small lymphocytes were also positive for CD3, CD56, and TIA-1 stains and negative for CD20. EBER Epstein-Barr virus-encoded small RNA, H/E hematoxylin and eosin 


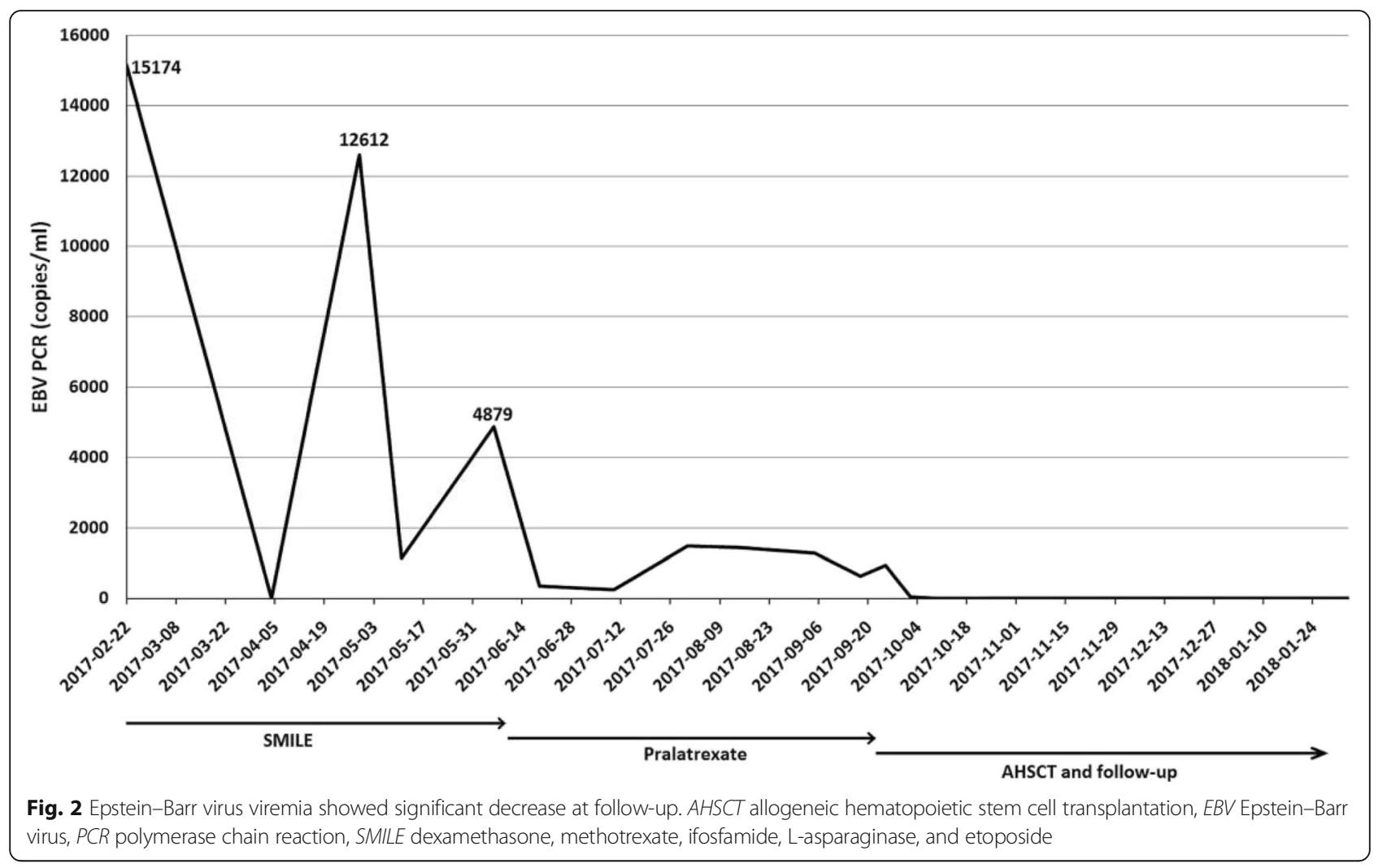

December 2018. The skin lesion revealed good healing without progression. We also gave low-dose nivolumab $(20 \mathrm{mg})$ every month for maintenance therapy until January 2020. A follow-up of PET-CT, EBV viremia, skin lesion, and bone marrow showed no evidence of disease relapse.

\section{Discussion and conclusions}

The treatment for advanced-stage disseminated ENKL, nasal type, is very challenging for clinicians. Because ENKL cells are associated with a high expression of Pglycoprotein, which causes multidrug resistance and could contribute to the poor response associated with
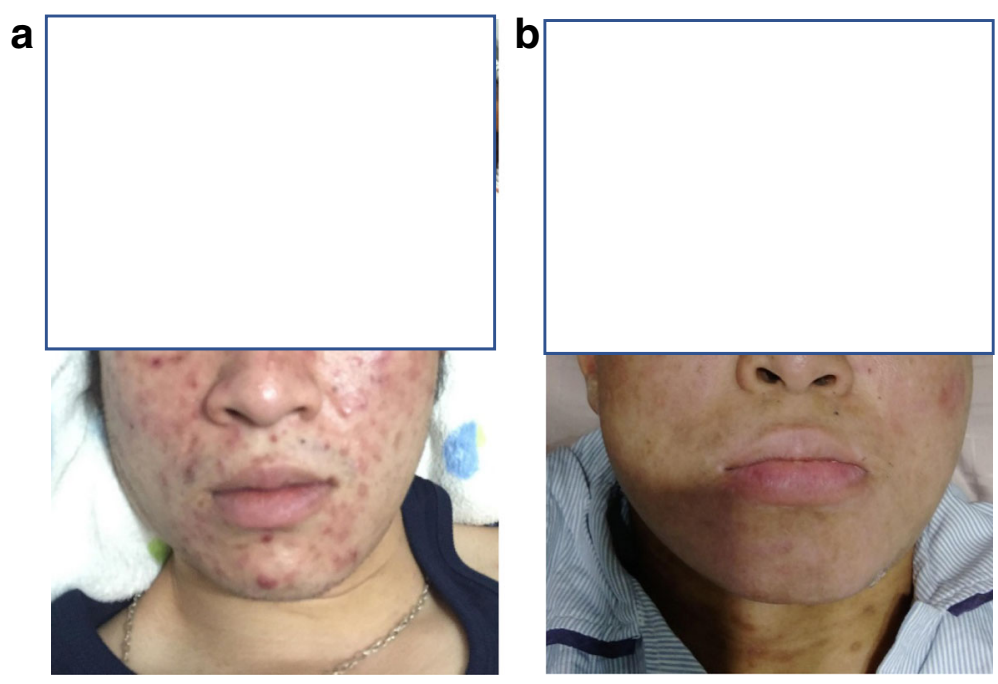

Fig. 3 Skin lesions of face and trunk at diagnosis (a) and after allogeneic hematopoietic stem cell transplantation (b) revealed complete remission 

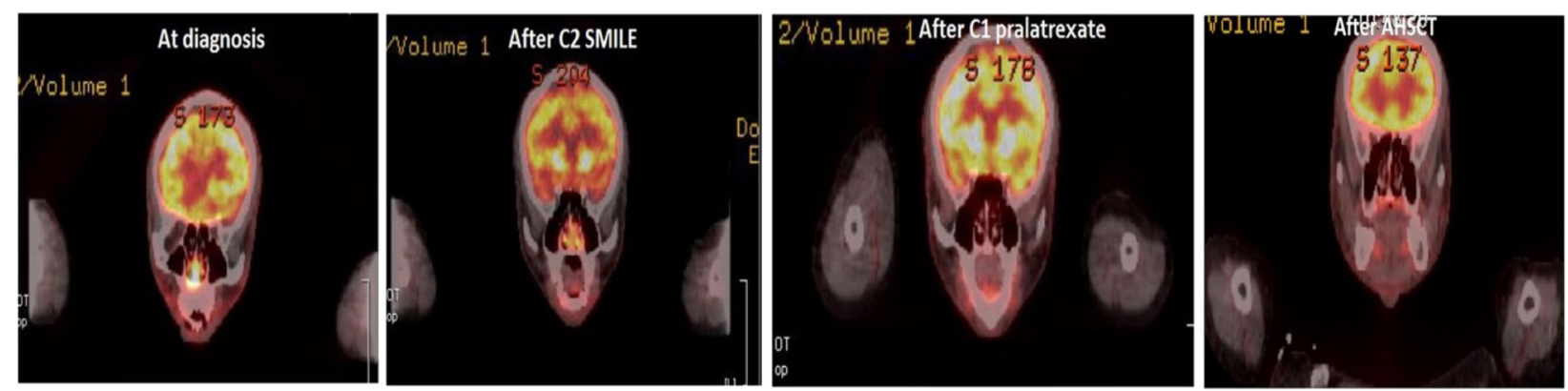

Fig. 4 Positron emission tomography-computed tomography scans for nasal lesions and lymphadenopathies showed complete metabolic response after one cycle of pralatrexate and maintained the response after allogeneic hematopoietic stem cell transplantation. AHSCT allogeneic hematopoietic stem cell transplantation, SMILE dexamethasone, methotrexate, ifosfamide, L-asparaginase, and etoposide

anthracycline-based chemotherapy regimens, we opted to use the SMILE regimen in our patient [8]. Once ENKL is deemed primary refractory to first-line chemotherapy, the prognosis is very poor $[5,6]$. Although AHSCT is a potentially curative therapy with the possibility of disease relapse prevention, salvage chemotherapy with an effective treatment as a bridge to AHSCT is not well understood, especially for advanced-stage nasal type ENKL. Most patients often die of disease progression, infections, or associated complications after failure of first-line chemotherapy.

Our patient received a total of two cycles of SMILE chemotherapy and was unfortunately considered primary refractory to this therapy. He demonstrated a potentially excellent therapeutic effect of PDX in a patient with stage IV ENKL, nasal type, refractory to first-line SMILE. Through a bridge of PDX, he ultimately underwent AHSCT, leading to complete remission of disease. This is the first presentation to explore PDX as a successful bridge to AHSCT in a patient with advanced-stage ENKL refractory to first-line SMILE chemotherapy.

Circulating EBV DNA levels have been observed to be important for prognostication in NKTCL [3]. The National Comprehensive Cancer Network (NCCN) now recommends monitoring EBV DNA load and calculation of Prognostic Index of Natural Killer Lymphoma (PINK) or Prognostic Index of Natural Killer Lymphoma with Epstein-Barr Virus DNA (PINK-E) as part of the initial workup for ENKL. These recommendations are based on a retrospective study that analyzed EBV DNA in 328 patients and determined that a detectable EBV DNA titer was an independent prognostic factor for overall survival (OS) [16].A prospective observational study in patients with NKTCL treated with an asparaginasebased therapy determined that pretreatment EBV DNA levels correlated with a lower rate of $C R$, and posttreatment EBV DNA levels were a prognostic factor for both progression-free survival (PFS) and OS (in patients with $\mathrm{CR}$, post-treatment EBV DNA positivity correlated with inferior PFS and OS; $p<0.0001)$ [17]. Similarly, another study found that higher levels of pretreatment EBV DNA predicted poor response rates to the SMILE regimen, indicating that the pretreatment EBV DNA level may be a positive predictive marker for response after asparaginase-based treatment [18]. If these findings are validated in future prospective clinical trials, patients with high pretreatment EBV DNA levels may benefit from more intensive treatments or novel drugs other than asparaginase-based therapy. In our patient, the EBV viral load rebounded during failure to SMILE with progressive generalized symptoms and skin lesions, but showed a significant downregulation of plasma EBV viral titer from the point of PDX therapy. An undetectable plasma EBV viral titer was noticed after AHSCT. The improvement of EBV viremia in our patient was also compatible with continuous clinical disease control.

In summary, our case demonstrates the successful use of PDX as a successful bridge to AHSCT, which played an integral role in our patient's overall outcome, leading to complete disease remission. Due to the rarity of ENKL, it is unlikely that randomized trials will be conducted; however, based on our case's outcome, we believe that this therapeutic schema warrants further evaluation to expand treatment options available to patients with advanced relapsed/refractory nasal type ENKL.

\footnotetext{
Abbreviations

aGVHD: Acute graft-versus-host disease; AHSCT: Allogeneic hematopoietic stem cell transplantation; CCRT: Concurrent chemoradiotherapy;

CR: Complete response; CT: Computed tomography; EBER: Epstein-Barr virusencoded small RNA; EBV: Epstein-Barr virus; ENKL: Extranodal natural killer/T cell lymphoma; FDG: Fluorodeoxyglucose; GeLOX: Gemcitabine, Leunase (Lasparaginase), and oxaliplatin; HSCT: Hematopoietic stem cell transplantation; NCCN: National Comprehensive Cancer Network; NK: Natural killer; NKTCL: Natural killer/T cell lymphoma; OS: Overall survival; PCR: Polymerase chain reaction; PDX: Pralatrexate; PET: Positron emission tomography; PFS: Progression-free survival; PINK: Prognostic Index of Natural Killer Lymphoma; PINK-E : Prognostic Index of Natural Killer Lymphoma with Epstein-Barr Virus DNA; PTCL: Peripheral T cell lymphoma; SMILE: Dexamethasone, methotrexate, ifosfamide, L-asparaginase, and etoposide
} 


\section{Acknowledgements}

Medical writing assistance was provided by Phillips Gilmore Oncology Communications, Inc. (Philadelphia, PA, USA).

\section{Authors' contributions}

Yao-Chung Liu was the principal investigator, collected data, and generated the study design, figures, tables, and manuscript. Hao-Yuan Wang cared for the patient and applied for the informed consent of the patient. Jyh-Pyng Gau, TingAn Lin, Chia-Jen Liu, Sheng-Hsuan Chien, and Liang-Tsai Hsiao helped conceive the study and participated in manuscript preparation. Yao-Chung Liu performed medical chart reviews. All authors participated in data interpretation and critical review of the final paper and submission. The authors read and approved the final manuscript.

\section{Funding}

Financial support for medical translation and writing was provided by Mundi Pharma.

\section{Availability of data and materials}

The datasets used and/or analyzed during the current study are available from the corresponding author on reasonable request.

\section{Ethics approval and consent to participate}

Written informed consent for publication of this paper was obtained from the patient.

\section{Consent for publication}

Written informed consent was obtained from the patient for publication of this case report and any accompanying images. A copy of the written consent is available for review by the Editor-in-Chief of this journal.

\section{Competing interests}

The authors declare that they have no competing interests.

\section{Author details}

'Division of Haematology, Department of Medicine, Taipei Veterans General Hospital, No. 201, Section 2, Shipai Road, Beitou District, Taipei City 112, Taiwan. ${ }^{2}$ Faculty of Medicine, National Yang-Ming University, No. 155, Section 2, Linong St, Beitou District, Taipei City 112, Taiwan. ${ }^{3}$ Division of Transfusion Medicine, Taipei Veterans General Hospital, No. 201, Section 2, Shipai Road, Beitou District, Taipei City 112, Taiwan.

\section{Received: 24 October 2019 Accepted: 20 February 2020}

\section{Published online: 17 March 2020}

\section{References}

1. Swerdlow SH, Campo E, Harris NL, et al. World Health Organization Classification of Tumors of Haematopoietic and Lymphoid Tissues. Lyon: IARC Press; 2008. p. 285-8.

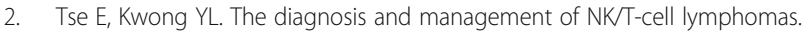
J Hematol Oncol. 2017;10:85.

3. Au WY, Pang A, Choy C, Chim CS, Kwong YL. Quantification of circulating Epstein-Barr virus (EBV) DNA in the diagnosis and monitoring of natural killer cell and EBV-positive lymphomas in immunocompetent patients. Blood. 2004;104:243-9.

4. Cai Q, Cai J, Fang Y, Young KH. Epstein-Barr virus-positive natural killer/T-cell lymphoma. Front Oncol. 2019;9:386.

5. Lee J, Park YH, Kim WS, et al. Extranodal nasal type NK/T-cell lymphoma: elucidating clinical prognostic factors for risk-based stratification of therapy. Eur J Cancer. 2005:41:1402-8.

6. Chim CS, Ma SY, Au WY, et al. Primary nasal natural killer cell lymphoma: long-term treatment outcome and relationship with the International Prognostic Index. Blood. 2004;103:216-21.

7. Kwong YL, Kim WS, Lim ST, et al. SMILE for natural killer/T-cell lymphoma: analysis of safety and efficacy from the Asia lymphoma study group. Blood. 2012;120:2973-80

8. Yamaguchi M, Kwong YL, Kim WS, et al. Phase II Study of SMILE chemotherapy for newly diagnosed stave IV, relapsed, or refractory extranodal natural killer (NK)/T-cell lymphoma, nasal type: the NK-cell tumor study group study. J Clin Oncol. 2011;29:4410-6.
9. Ahn HK, Kim SJ, Hwang DW, Ko YH, Tang T, Lim ST, Kim WS. Gemcitabine alone and/or containing chemotherapy is efficient in refractory or relapsed NKT-cell lymphoma. Investig New Drugs. 2013;31:469-72.

10. Kim WY, Nam SJ, Kim S, Kim TM, Heo DS, Kim CW, Jeon YK. Prognostic implications of CD30 expression in extranodal natural killer/T-cell lymphoma according to treatment modalities. Leuk Lymphoma. 2015;56:1778-86.

11. Hui J, Przespo E, Elefante A. Pralatrexate: a novel synthetic antifolate for relapsed or refractory peripheral T-cell lymphoma and other potential uses. J Oncol Pharm Pract. 2012;18:275-83.

12. O'Connor OA, Pro B, Pinter-Brown $L$, et al. Pralatrexate in patients with relapsed or refractory peripheral T-cell lymphoma: results from the pivotal PROPEL study. J Clin Oncol. 2011;29:1182-9.

13. Tse E, Chan TS, Koh LP, et al. Allogeneic haematopoietic SCT for natural killer/T-cell lymphoma: a multicentre analysis from the Asia Lymphoma Study Group. Bone Marrow Transplant. 2014;49:902-6.

14. Kanate AS, DiGilio A, Ahn KW, et al. Allogeneic haematopoietic cell transplantation for extranodal natural killer/T-cell lymphoma, nasal type: a CIBMTR analysis. Br J Haematol. 2017;182:916-20

15. Kharfan-Dabaja MA, Kumar A, Ayala E, et al. Clinical practice recommendations on indication and timing of hematopoietic cell transplantation in mature T cell and NK/T cell lymphomas: an international collaborative effort on behalf of the Guidelines Committee of the American Society for Blood and Marrow Transplantation. Biol Blood Marrow Transplant. 2017;23:1826-38.

16. Kim SJ, Yoon DH, Jaccard A, et al. A prognostic index for natural killer/T-cell lymphoma after non-anthracycline-based treatment: a multicenter, retrospective analysis. Lancet Oncol. 2016;17:389-400.

17. Wang $L$, Wang $H$, Wang $J$, et al. Post-treatment plasma EBV-DNA positivity predicts early relapse and poor prognosis for patients with extranodal NKVT cell lymphoma in the era of asparaginase. Oncotarget. 2015;6:30317-26.

18. Kwong YL, Pang AW, Leung AY, Chim CS, Tse E. Quantification of circulating Epstein-Barr virus DNA in NKT-cell lymphoma treated with the SMILE protocol: diagnostic and prognostic significance. Leukemia. 2014;28:865-70.

\section{Publisher's Note}

Springer Nature remains neutral with regard to jurisdictional claims in published maps and institutional affiliations.

Ready to submit your research? Choose BMC and benefit from:

- fast, convenient online submission

- thorough peer review by experienced researchers in your field

- rapid publication on acceptance

- support for research data, including large and complex data types

- gold Open Access which fosters wider collaboration and increased citations

- maximum visibility for your research: over $100 \mathrm{M}$ website views per year

At BMC, research is always in progress.

Learn more biomedcentral.com/submission 